\title{
4 To Synthesize and Evaluate Using Assessment Tools
}

\subsection{Introduction}

As explained in Section 1.2.1, the focus of the fifth and sixth levels of Bloom's taxonomy is on synthesizing and evaluating new knowledge. In this chapter, my goal is to tell you about several resources that you may find useful to achieve goals in these levels. See Figure 4.1 for a general overview of the chapter.

\section{- delicious Web'Notes}

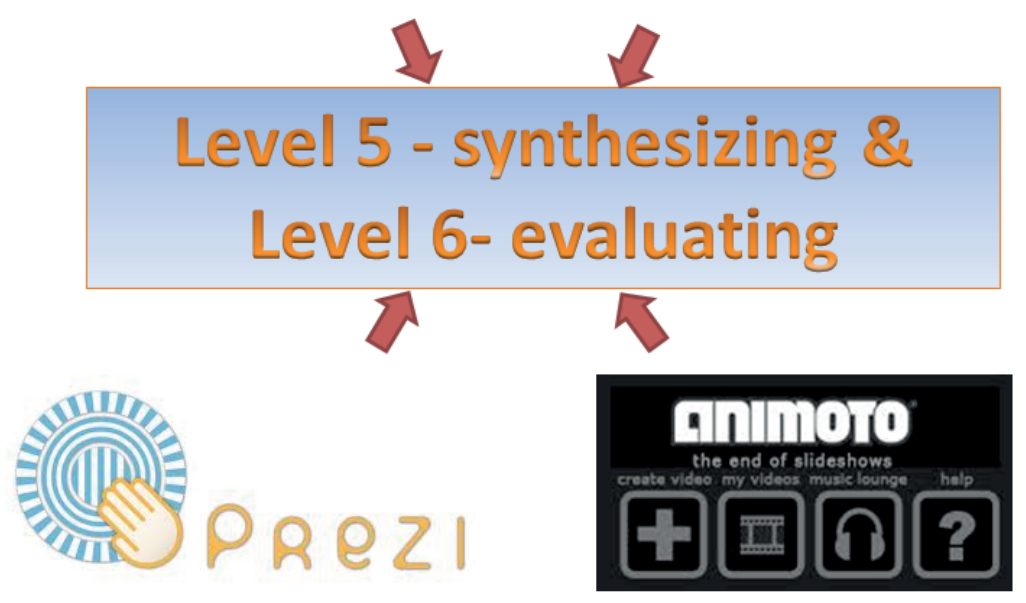

Figure 4.1: General overview of chapter 4 (tools for the levels 5 \& 6 of Bloom's taxonomy)

The resources described in this chapter have been chosen because, from my experience, I have had the opportunity of checking that students are able to use them, and that they are tools that serve to accomplish the formulated pedagogical goal. Another reason is that they are free, and I can show you how to use them step-by-step.

As you get familiar with these tools, I am sure you will be able to find others that you can find that are even more useful for your particular case. Moreover, you will be able to create your own resources. So, let's start with the possibilities of Delicious in Section 4.2, Webnotes in Section 4.3, Prezi in Section 4.4, and Animoto in Section 4.5. The chapter ends, as always, with several solved exercises. Remember that this book is not a novel, and that you must practice what you read in order to take advantage of it! 


\subsection{Delicious}

Delicious allows you to manage on-line bookmarks (i.e. webpages that you consider interesting and would like to use from any device connected to the Internet). Let's think about this situation. You have just found an incredible webpage with thousands of resources. So, you click on your browser to save it as a bookmark. The next day, you go to your school, and you want to share this incredible webpage with your colleagues. What happens? Yes, no way of doing that! The webpage has been saved on your computer at home, so you need to remember it if you would like to access that webpage on the computer at school. Delicious gives you the possibility of saving the bookmarks so that you can access them from any computer connected to the Internet.

Moreover, in Delicious, bookmarks are tagged so that they can be found in a more effective way. OK, but how does it relate with the $5^{\text {th }}$ and $6^{\text {th }}$ levels of Bloom's taxonomy? Well, teachers are not the only ones who can find interesting webpages and save them as bookmarks. When we go to the upper levels of Bloom's taxonomy, the idea is that students are responsible for their knowledge, they can become creators of new knowledge, and they should be able to evaluate and criticize the information gathered. Delicious is a wonderful tool for your students to explore the web of bookmarks saved by other people, and save their own bookmarks. Moreover, they can share their bookmarks with other students, and teachers too!

Does it seem interesting? I hope so! Let's start using Delicious ${ }^{33}$. To do that, the first step is to register and get an account. As you can see in Figure 4.2, the welcome page is pretty simple.

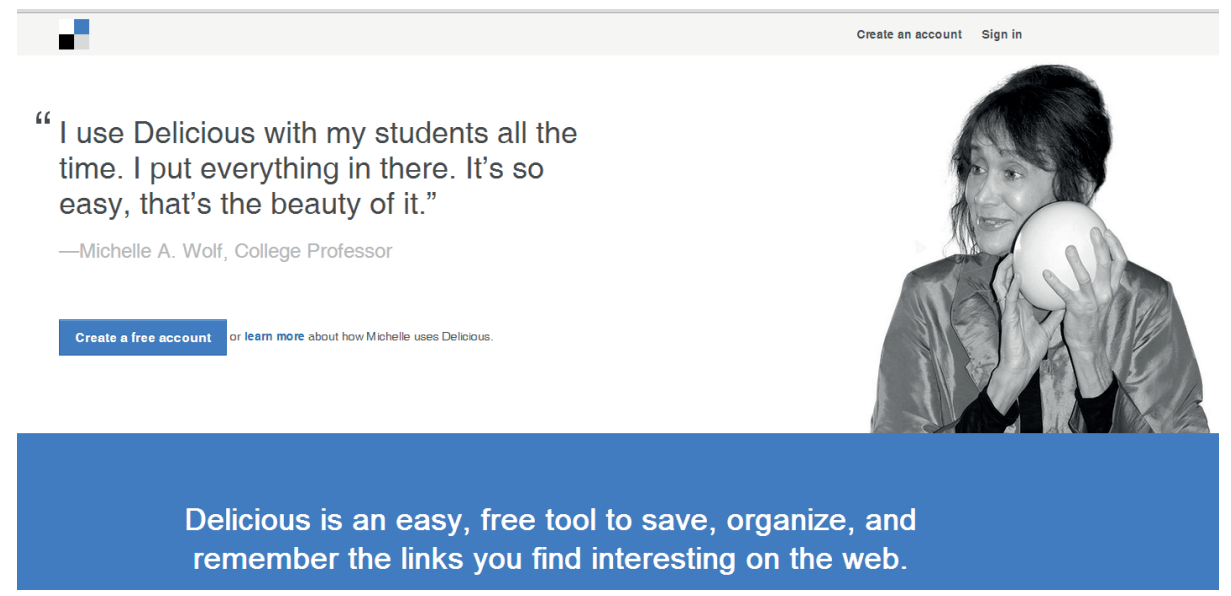

Figure 4.2: Welcome Delicious webpage

33 https://delicious.com/ 
The two most important buttons are on the upper right corner. There, you can sign in if you have an account, or create a new one. If you just want to explore the webpage, you can scroll down a little, and start exploring links, but you cannot save them unless you have an account and you have signed in. It is also possible to use an already existing account from a social network, such as Facebook or Twitter, instead of creating a new account. That way, you just enter with one of your existing usernames and password. Otherwise, you click on the "Create an account" button (you can see it on the upper right corner of Figure 4.2) and fill in a simple form similar to the one shown in Figure 4.3

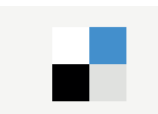

Create a new Delicious account.

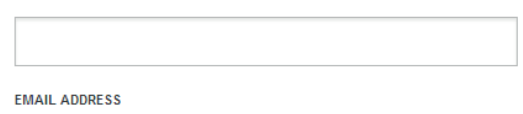

EMAIL ADDRESS

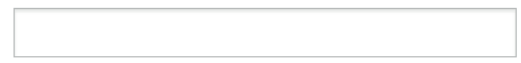

FULL NAME

Example: Lauren Ipsum

PASSWORD

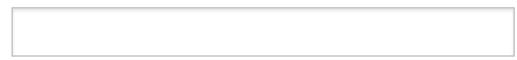

$\square$ Check Password

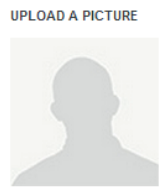

Seleccionar archivo No se ha seleccionado ningún archivo

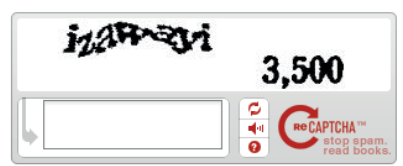

\section{Create my account}

By clicking "Create my account" you are indicating that you have read and agree to the Terms of Service.

Figure 4.3: Creating an account in Delicious (I)

You can also upload a photo, and do not forget to write the letters and numbers of the kaptcha before clicking on the "Create my account" or "Join Delicious" (the text could change) blue button at the bottom. That's it! Now you have your account in Delicious and you can start saving and sharing bookmarks. The webpage that I got was similar to the one shown in Figure 4.4. 


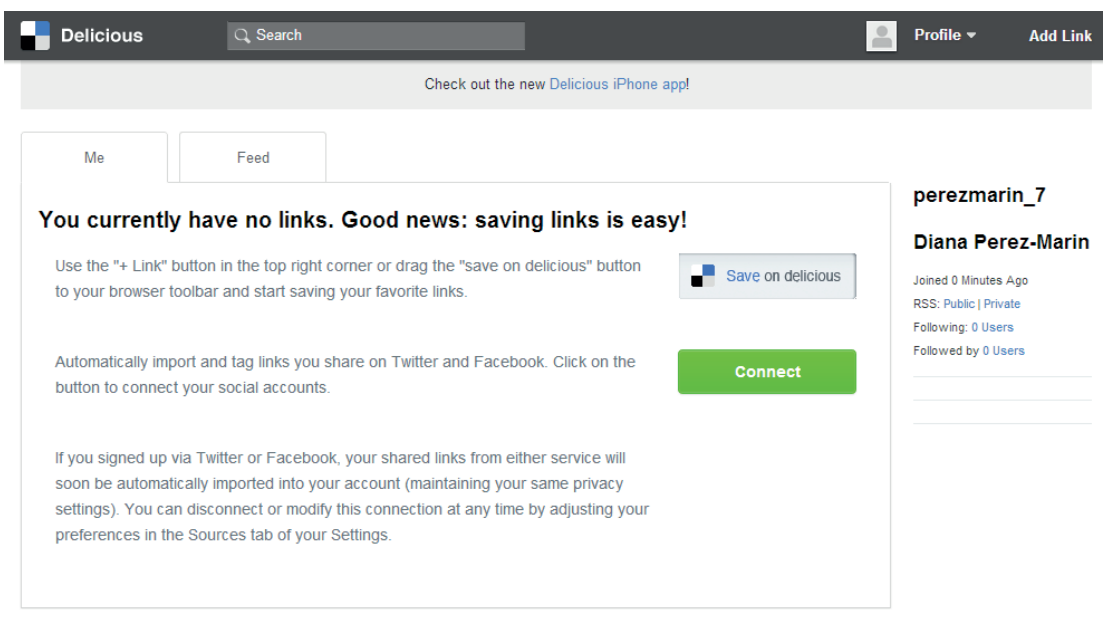

Figure 4.4: Creating an account in Delicious (II)

As you can see, initially there are no bookmarks, your account is empty! However, adding a link is really easy. Can you see on the upper right corner (yes, very important corner!) the "Add link" text? If you know the bookmark that you want to save, you just need to click on "Add link" and you save it. For instance, if I want to save coursera.org, the repository of MOOCs that I talked about in Section 2.3, then I get a page similar to the one shown in Figure 4.5

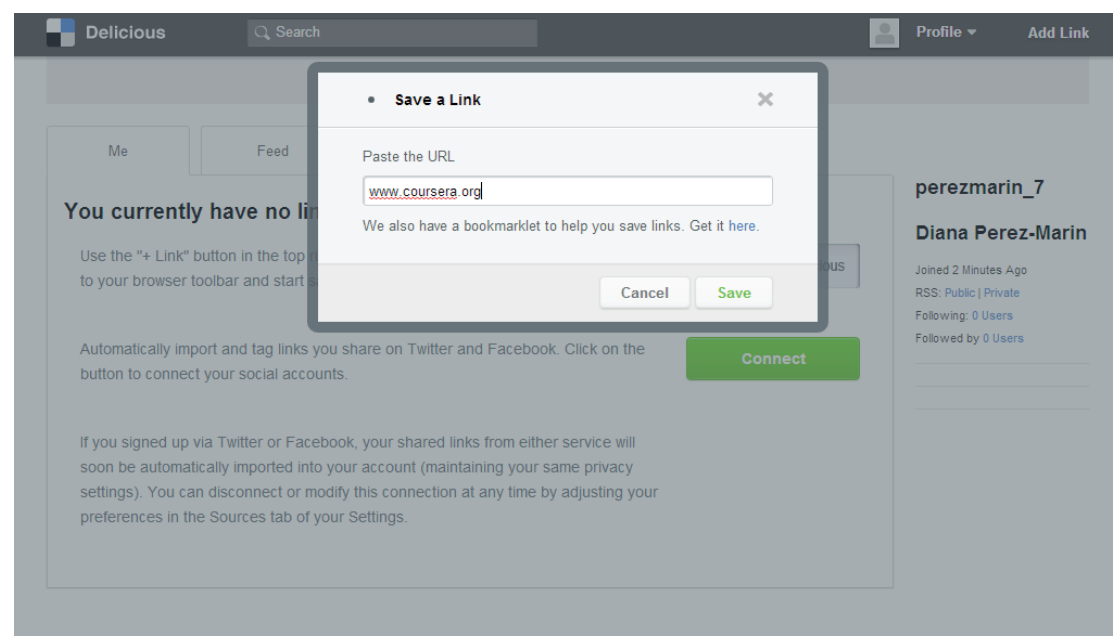

Figure 4.5: Saving a bookmark on-line in Delicious (I) 
As you may remember, I have told you that one good feature of the bookmarks in Delicious is that you can tag them (i.e. you can classify them by providing a label and / or additional information like a description or a photo). Where can you do that? Well, when you click on the "Save" button, the next page that you see is where you can provide more information (it is not necessary but it is usually useful!). Figure 4.6 shows you this page.

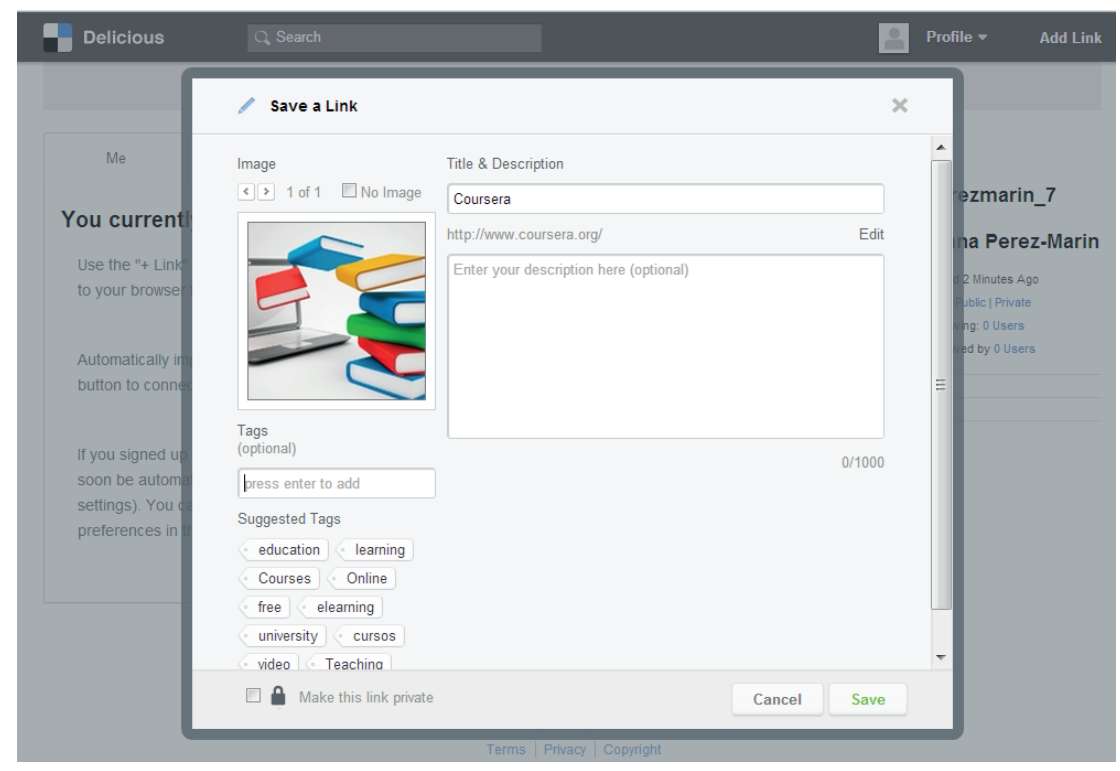

Figure 4.6: Saving a bookmark on-line in Delicious (II)

You can help yourself with the suggested tags, and the images provided by Delicious. By default, all bookmarks will be public (the idea is to share!). However, if you want the bookmark to be private, you can click on the "Make this link private" checkbox on the lower left corner. Now, you can click on the "Save" button to see how your first bookmark has been saved in your account. Figure 4.7 shows how the Delicious page changes now that there is a bookmark.

Let's look in a bit more detail at the bookmark you saved. As you can see, there is the name that you provided at the top (Coursera), on the right the image, and below a number, a link and several tags. You can edit the name, link and tags of the bookmark at any time just by clicking on the "pen" icon. On the other hand, you cannot edit the number of saves. Delicious counts how many people share the same bookmark. This "saves" counter is a good indicator of the popularity of the bookmark. Usually, bookmarks with many saves are interesting bookmarks! 


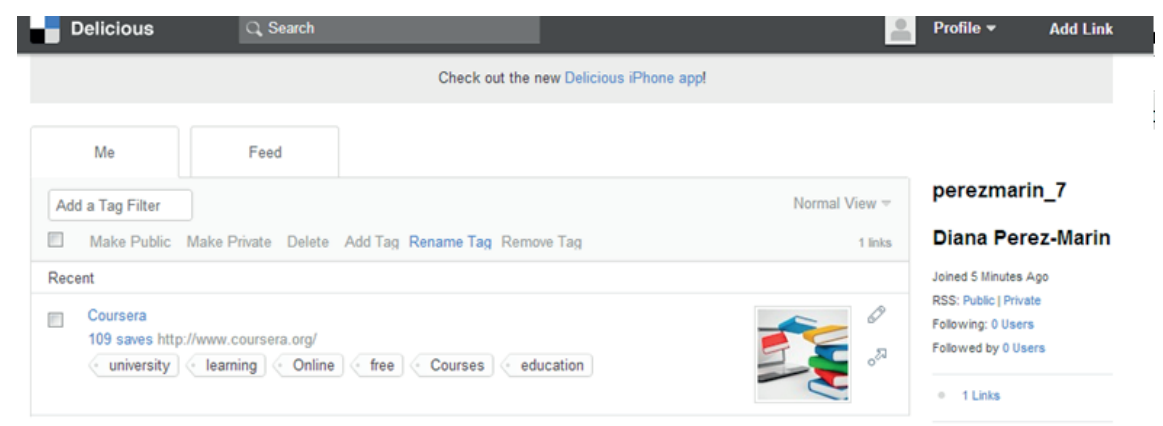

Figure 4.7: Sample bookmark saved in Delicious

What happens if the teacher wants their students to save five bookmarks regarding pregnancy? Students must search! They do not have the links, so they cannot directly type them as in the previous sample. They can search in Delicious or, on the Internet. To search in Delicious, you can use the search engine that is at the top of the page (see Figure 4.7) and type the keywords (as in Google). If you type "pregnancy" you will see a page similar to the one shown in Figure 4.8

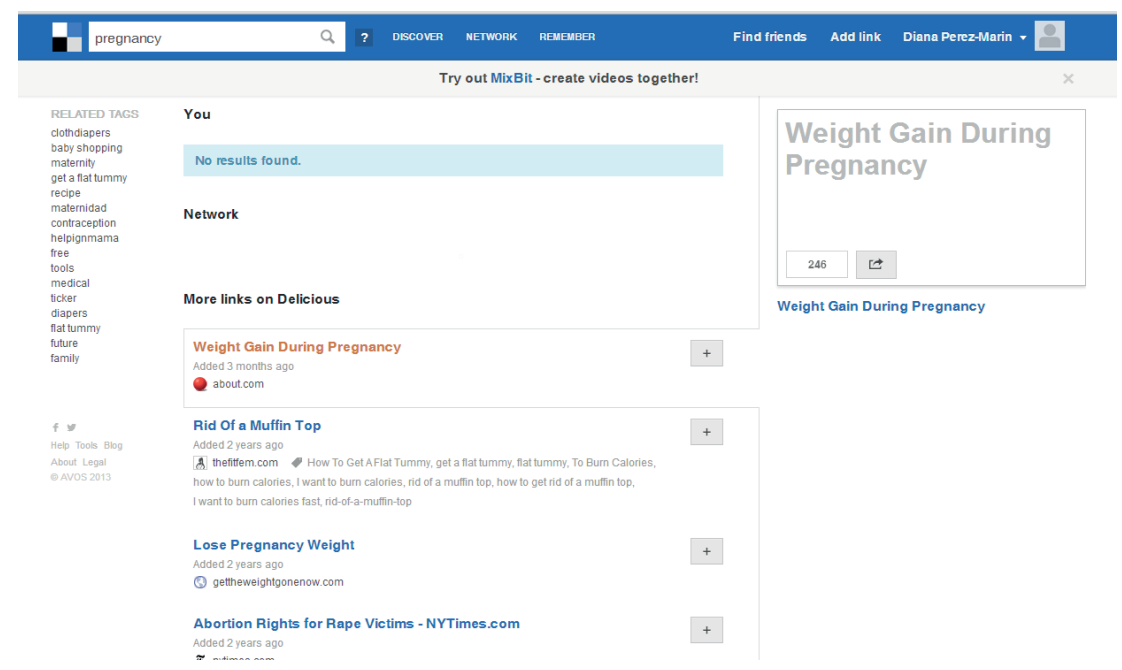

Figure 4.8: Results webpage of a search in Delicious

The results are classified according to what bookmarks were found among the ones that you saved (currently, none because we have not saved anything about that topic yet), the ones that your friends (your network) saved (with the idea that your friends usually have similar interests to you), and the bookmarks collected by everyone on 
Delicious (here, you will always find bookmarks, although you must look at them carefully to make sure that they are what you are looking for).

When you find an interesting link, you can save it just by clicking on the "+" button right next to it. If you want to see your bookmarks, you can click on the "Remember" text at the top, or you can click on your name. If you click on the bookmark, you can go to the webpage and if you find that you do not want to keep this bookmark any longer, you just need to click on the pen icon, as if you were going to edit it, but then you click on "Remove link" (to the left of the "Cancel") and the blue button to "Save changes". It is now time for you to explore Delicious, and to think how you can use it for your students to reach levels 5 and 6 in Bloom's taxonomy (you can also use it just to show a video, levels 1 or 2 of Bloom, or a fun activity for levels 3 or 4). Do not forget to click on your username and "Sign out" before leaving the webpage (just closing it is not enough), and sign in every time that you want to use Delicious from any device connected to the Internet, and enjoy using it!

\subsection{Webnotes}

If Delicious is very useful for sharing and saving bookmarks, Webnotes ${ }^{34}$ is the equivalent for notes in webpages. I'll explain that a bit more. Sometimes, you do not need or want to save the whole webpage, because you are only interested in one or two paragraphs. Wouldn't it be wonderful just to save those paragraphs, with the reference to the source webpage? It's a dream for any researcher, and in general, for anyone (say student or teacher) who needs to gather information about a topic, maybe in order to produce a report or evaluate something from a critical point of view. Figure 4.9 shows the Webnotes main page with an example of a web page that has been annotated, so that you can see what I am talking about.

Webnotes has three versions depending on the price. Oh...so do I have to pay to use Webnotes? Well, no. Remember that I told you I would only choose free tools, which I have seen that are easy to use? Webnotes has a lite ${ }^{35}$ free version that allows you to annotate webpages, organize and search notes, and share notes via email, twitter and permalink (i.e. a link that does not change, so that you can make sure that you can come back and the link will be working).

$34 \mathrm{http}: / /$ www.webnotes.net/

35 A lite (light) version of commercial computer software is usually provided free, but it does not have full functionality, and/or it is supported by advertisements. Moreover, lite versions do not usually have tech support. 


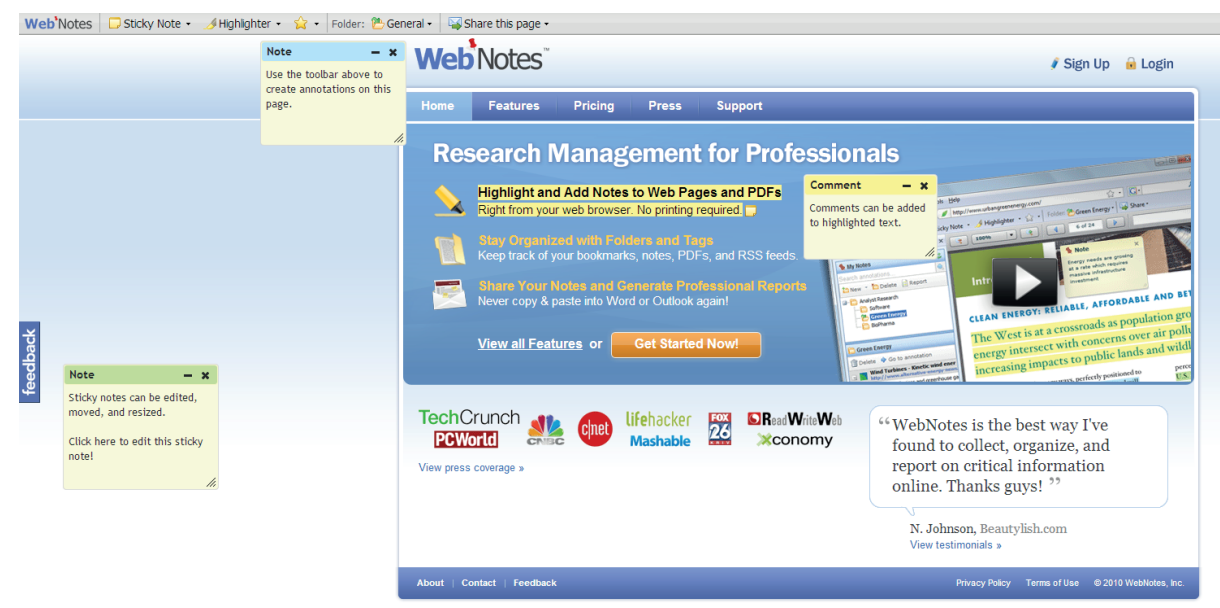

Figure 4.9: Sample annotated webpage (in particular, the Webnotes webpage)

Does it seem interesting? I hope so! So, let's start using Webnotes. To do that, given that it is an on-line application you do not need to install anything on your computer, but you need to create an account as usual. The good thing it is really simple. You click on Sign Up (where do you think it is? Yes, it is in the upper right corner! Well done!), and next, on the "Sign Up" orange button in the "Lite" column for the free version of the tool. It is possible that there is some kind of promotion, so that even if you click on the Lite version, they upgraded your account to the Pro version for a limited amount of time, but I would not get used to that if you are not willing to pay later, because it will not last. This is why I would just stick to what is available in the free version. See in Figure 4.10 how to sign up in the Lite version of Webnotes. When you click on the "Sign up" orange button, you need to complete the steps and fill in the form with the basic information to set up the account. It is very important that in the second step you drag the "Annotate" button to your browser toolbar if you want to access Webnotes directly by clicking on your browser's toolbar button.

After that, you can click on the "Getting started" orange button to set up Webnotes in your browser. If your browser is Google Chrome, you can download the extension. Otherwise, you can just use the "Annotate" button that you dragged to your bookmarks toolbar. Webnotes will also offer a tutorial about how to create and save annotations, annotate PDFs, organize notes, share annotated pages and generate reports. You should now have a page similar to the one shown in Figure 4.11. If not, check the previous steps. Let's learn more about Webnotes with a step-by-step exercise! 


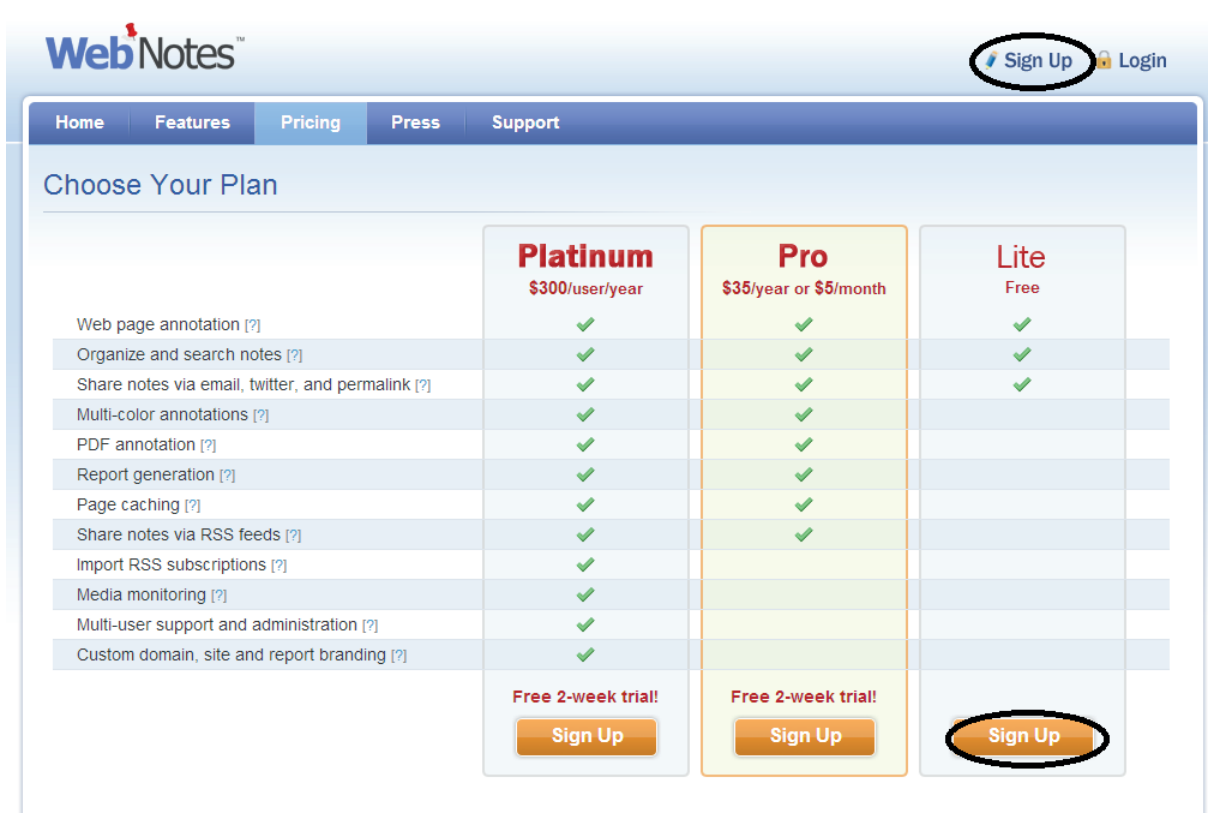

Figure 4.10: Webnotes sign up webpage

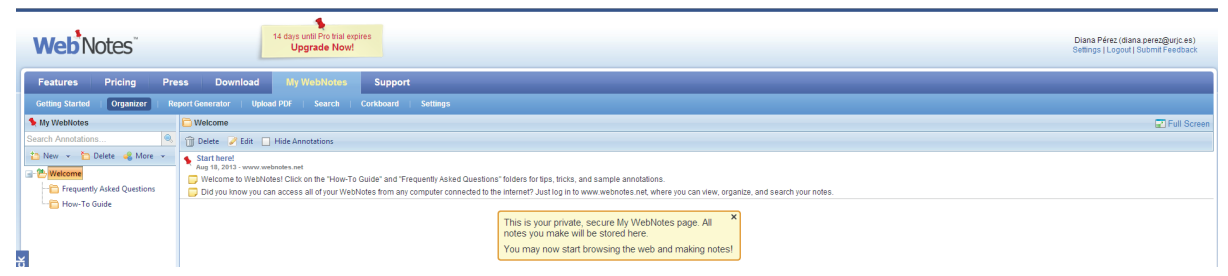

Figure 4.11: Webnotes Welcome page

Let's imagine that I am a student, and my teacher has asked me to write a report about the Battle of Achelous. I go on-line to search for information, and among other pages, I find a Wikipedia webpage ${ }^{36}$. I want to get some information and create some notes. To do that, I use Webnotes!

First step. To create and save annotations in a webpage and/or PDFs. When you are in your browser, if it is Google Chrome, and you have installed the extension, you just need to click on the red tack. Otherwise, you click on the Webnotes bookmarklet.

36 http://en.wikipedia.org/wiki/Battle_of_Achelous_(917) (use it, if you want to do the activity with me, step by step) 
Anyway, you have a menu bar similar to the one shown in Figure 4.12. As you can see, you can use sticky notes (like post-its), a highlighter, and you can also create a folder so that all your annotations are saved. If you cannot find it, check that you dragged the "Annotate" button when you signed up. You can read more information about how to access Webnotes at:

http://www.webnotes.net/Register/Register3.aspx?tool=bookmarklet.

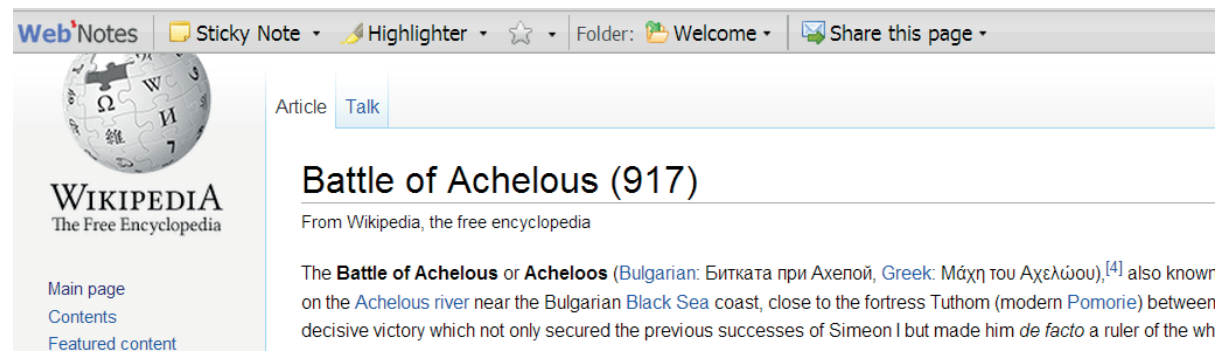

Figure 4.12: Webnotes menu bar

Let's create a folder called "The Battle of Achelous" by clicking in Folder $\rightarrow$ "New Folder". Then, create a sticky note with the text: "I have found the background part of this webpage very interesting, use that for Section 1.2 of the report!" by clicking on "Sticky note" and typing. Highlight the first sentence of the background, by clicking on the Highlighter and selecting the sentence (you can click on the note to delete it, and on the sentence and modify the background color). The annotations are shown in Figure 4.13.

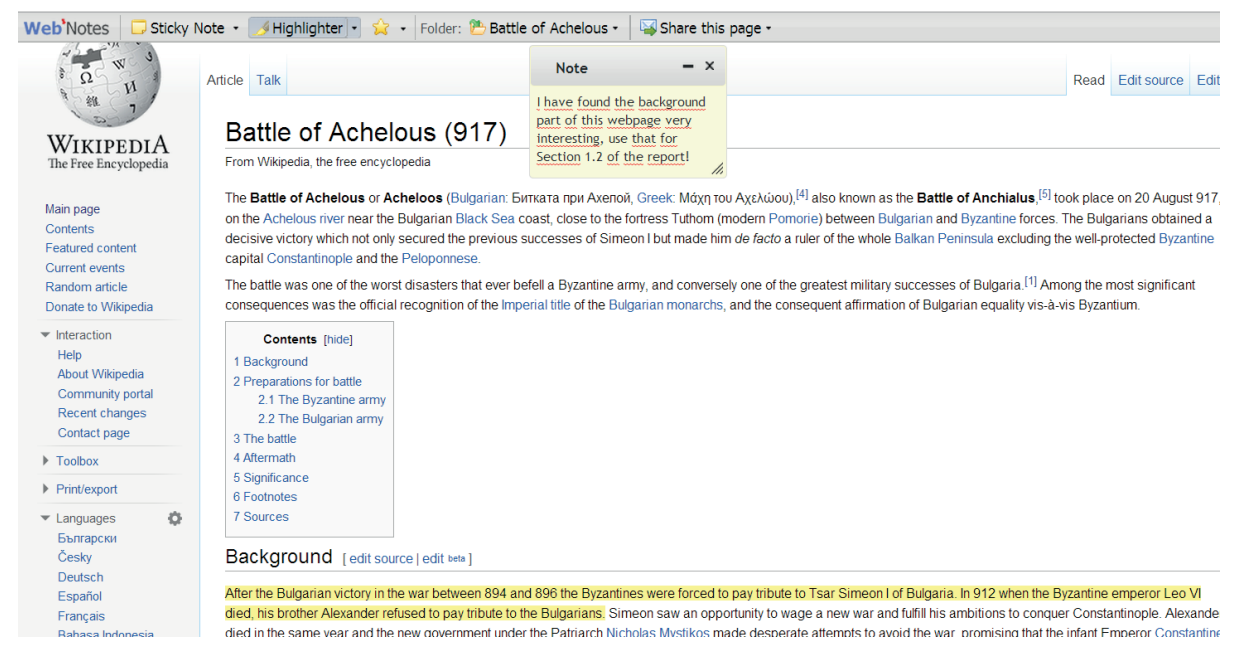

Figure 4.13: Sample annotated webpage using Webnotes 
I may also have found an interesting PDF when searching the web. I would also like to annotate it. How can I do that? You need to upload it to your Webnotes account. You can do that by clicking on the "Upload PDF" button (see Figure 4.11). The process is automatic, although it may take some time to convert it to a format in which Webnotes can annotate the PDF. When the process is finished, you will see the message "PDF Uploaded Successfully". Click on the button "Open with Webnotes”. Now you can also add notes and highlight sentences and save all the annotations in your folder.

I am going to write the note "Reference for this PDF: History of the Byzantine empire, A.A. Vasiliev, I need to find the year and editorial too!” (the procedure is the same as if I were adding a note in a webpage), and I am going to highlight the main sentence talking about the battle. See Figure 4.14 for a look at the annotated PDF. Finally, I save these notes in the "Battle of Achelous" folder.

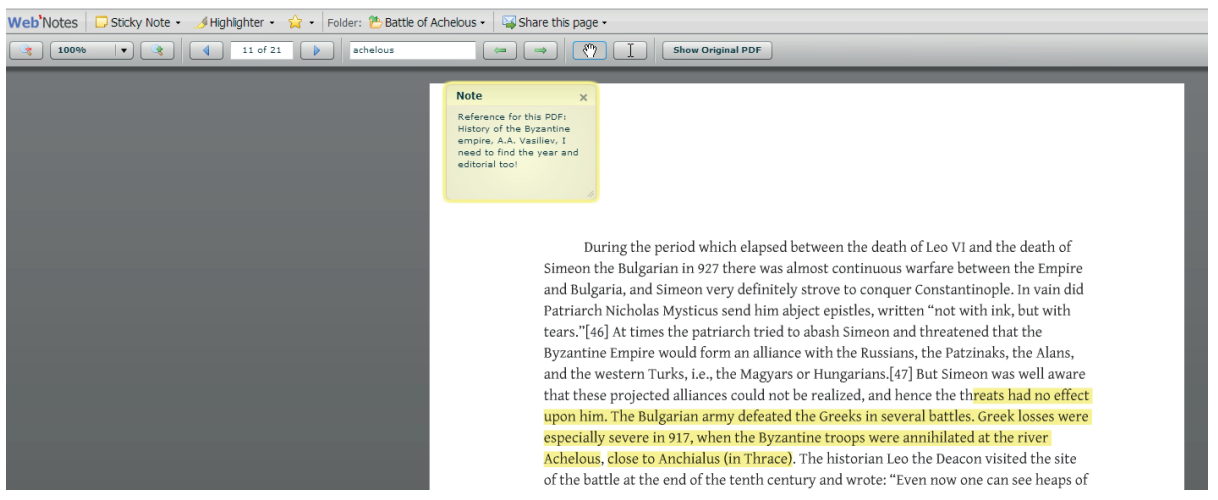

Figure 4.14: Sample annotated PDF using Webnotes

Second step. To organize your notes. OK, so now you have been collecting notes from different webpages and PDFs, and everything has been saved in the folder. Let's have a look at how it has been saved and how the Webnotes organizer works. If you click on the "Webnotes" icon in the upper left corner, you go to the main page, as shown in Figure 4.15.

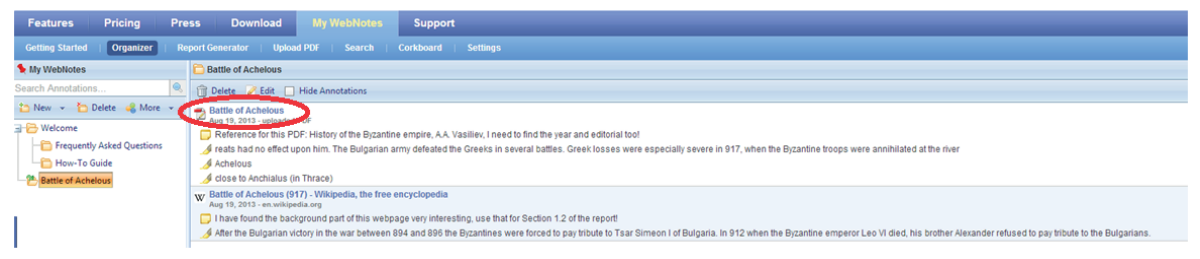

Figure 4.15: Organizer of Webnotes 
As you can see, each PDF or webpage annotated appears on the right (I have marked the PDF inside a red circle). Below, there is the date and the notes made from that source. If you click on the name (e.g. Battle of Achelous), you go back to the document just as you left it when you did the last annotation. You can also edit the annotations. For example, you can modify the title provided by clicking with the right button and choosing "Edit". Additionally, you can hide annotations or even delete them if you no longer find them useful. Moreover, what can happen if you start saving many annotations? That can become a mess! So, you have the possibility of creating subfolders, so you always keep a structure that is meaningful for you. To create a new subfolder, use the "New" button on the left panel. Finally, you can also search through the annotations. Figure 4.16 shows a results page when searching for "Achelous" in our step-by-step exercise.

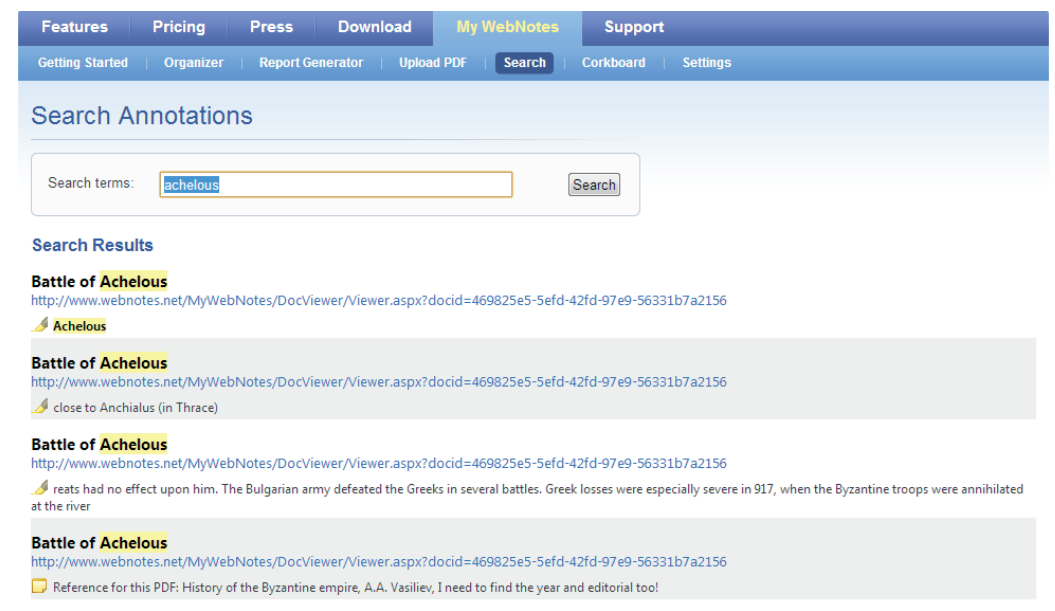

Figure 4.16: Results of a search in Webnotes

Third step (optional). To share your annotations. You can share the annotations made in both PDFs or webpages. This is really easy! You just click on the "Share this page" link on the upper menu (on the right) that you can see in Figure 4.12 (to share the annotations of a webpage) and Figure 4.14 (to share the annotations of a PDF). The possibilities for sharing are many: email, permalink, twitter, RSS and you can even generate a report to share, just by clicking on the "Report" option.

Fourth step. To generate a report. Finally, when you have finished saving all your annotations, it would be useful to have everything together in a report. It is possible to do that automatically with the "Report generator" in Webnotes. You click on "Report generator" (you can see it in Figure 4.16 between "Organizer" and "Upload PDF") and you'll have something similar to the page shown in Figure 4.17. 


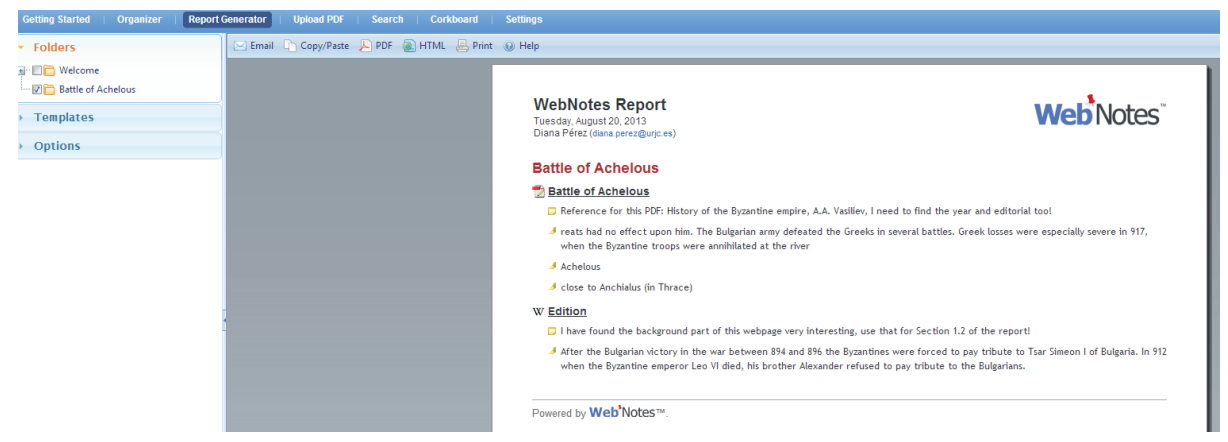

Figure 4.17: Report generated by Webnotes

This is the default report. On the left menu, you can click on "Templates" to change the appearance of the report. You can also click on "Options" to add a brief summary whilst you have a lot of information about these annotations in your mind, or add the link so that you do not forget the source. Moreover, if we look above the report, you can see that you can also download it as PDF by clicking on "PDF", or as a webpage by clicking on "HTML", print it or email it (by clicking on the "Print" option or "Email" option). OK, now you know the basics of Webnotes, I hope you have found it interesting. Please keep exploring the rest of the options in this tool and encourage your students to use it when researching. It can be really useful, and it saves time and paper! Do not forget to log out when you finish using your Webnotes account, and enjoy it!

\subsection{Prezi}

OK, we have finished our report, and we have uploaded it to Dropbox. Usually, at higher levels, a project does not end when the report is submitted, it is necessary to give an oral exposition. How can students give a good oral presentation? Well, you may remember from Section 2.4, that Google Drive has the option of creating presentations on-line. It even has presentation software, and it is a good choice, but it is not the only one. So, again, I would like to show you another way to create presentations, which has gained a lot of popularity in the last decades.

It is called "Prezi" ${ }^{37}$ and it allows you, in the free version of the tool, to create public presentations that are not based on the "slide" concept of traditional presentations, but on zooming in on the important concepts and their links. Imagine that you have all the space in the world, so you create a diagram and organize your ideas in it.

37 http://prezi.com/ 
You can show how the ideas are connected and talk more about particular parts by zooming in on certain aspects. In that case, it would be a good Prezi presentation. Are you interested in this other type of presentation? I hope so, because it really reaches the highest levels of Bloom's taxonomy since the student does not only have to search the web, and produce a report, but also has to organize their ideas in a good diagrammatic representation. Let's learn about Prezi. Figure 4.18 shows the Prezi welcome webpage with the sign up / login options in the upper right corner.

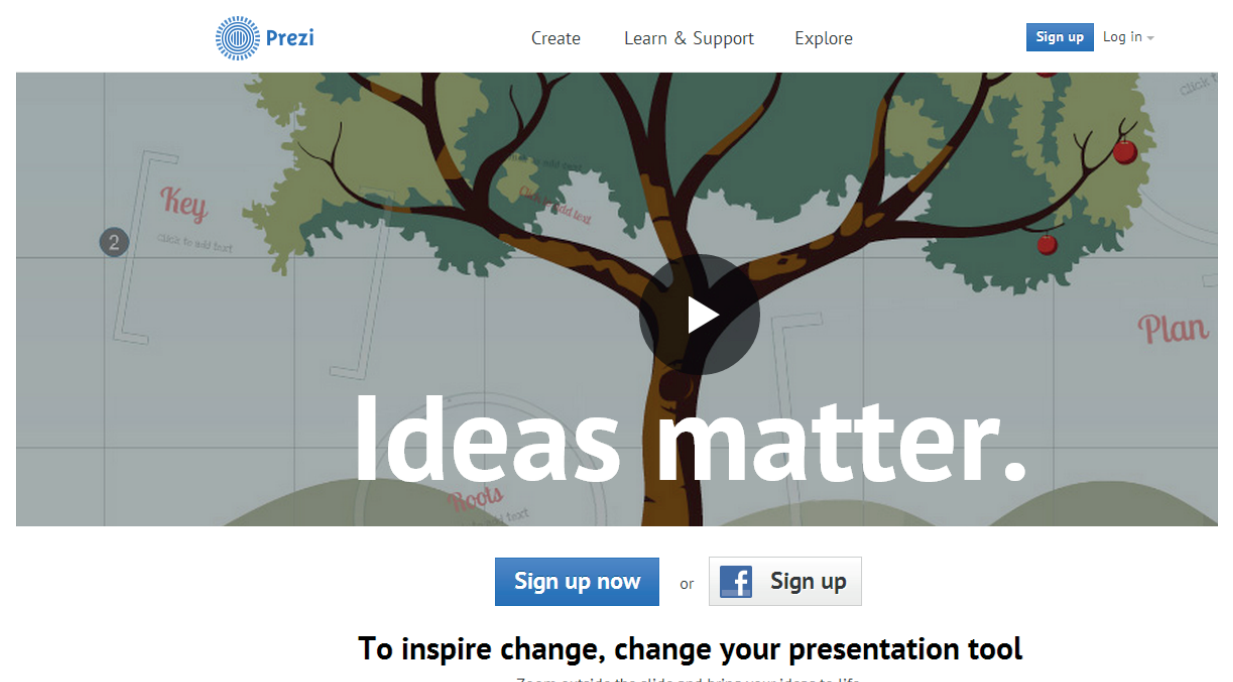

zoom outside the slide and bring your ideas to life.

Figure 4.18: Prezi welcome page

In the free version, you can create presentations on-line, although sometimes there are promotions in which, even with the free version, you can download the Windows desktop application for a limited amount of time. For now, let's create our first Prezi presentation on-line step-by-step, first by clicking on the "New prezi" button that you can see in Figure 4.19.

First step. Choose a template. As with other types of presentation, it is very important that it is something attractive to catch the attention of your audience. So, look at the templates provided, and depending on your goal, choose the one that you think is most appropriate by clicking on it, and next on the "Use template" button. Anyway, you can change it later in the edition page by clicking on "Themes". 


Your prezis Learn \& Support Explore Diana Perez -

\section{Refer 3 friends to Prezi and we'll upgrade your account for free! Get started ?}

ti New prezi

- All your prezis

P Owned by you

A Shared with you

Download Prezi for Windows

\pm Download 30 day free trial

- Did you know you can co-edit any prezi
All your prezis 0 preis $\cdot$ customize public profle

Search prezis

No prezis found.

Figure 4.19: Prezi main page

Second step. Edit the Prezi presentation. Once you have a template, you go to the edition page as shown in Figure 4.20. Currently, it is empty, so it is now time to complete it. You can start by providing a title, for instance, if the presentation is for the "Battle of Achelous", you can put that as title, something that attracts the attention and focuses on the topic. You can add frames and arrows, images, tables, etc. You can also share the presentations you create, either as a PDF or with a link.

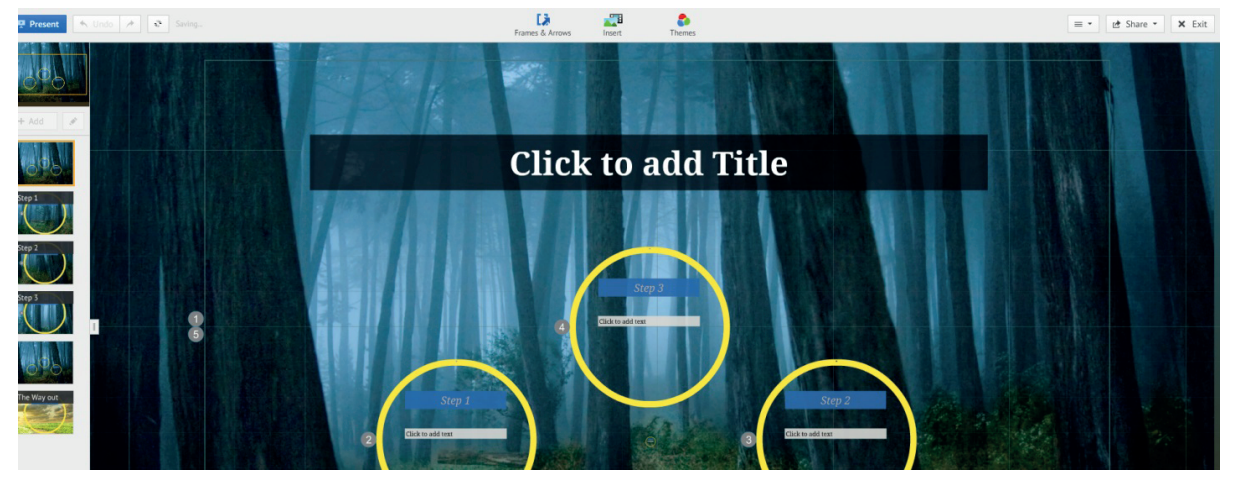

Figure 4.20: Prezi edition page 
You can see the creation of a Prezi presentation as an iterative process, following three steps: write, play and focus. First of all, you need to write your ideas, don't worry if they are not ordered. To write you double click at any place in the Prezi. When you see all your ideas in Prezi, it is usually easier to start creating connections in play mode. You can create hierarchies by making more important sentences bigger. Figure 4.21 shows the Prezi edition frame tool.

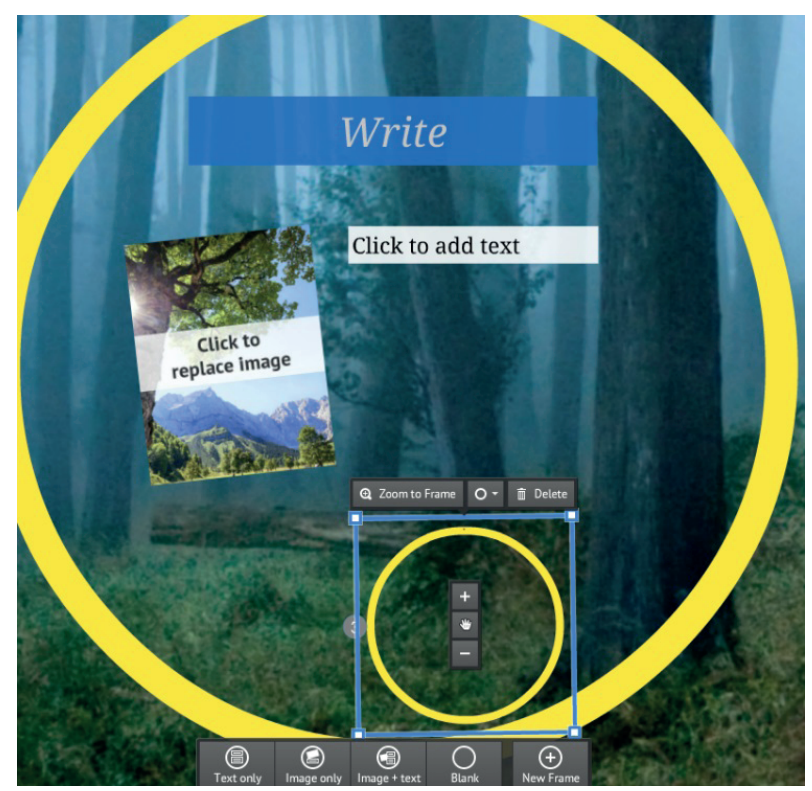

Figure 4.21: The edition frame tool

As you can see in the image, you can add new frames, and choose whether the frames will have text, images, or text and images. To edit the frame, you can click on "Zoom to Frame" and by clicking on the circle, you can empty the frame. Do not forget to save from time to time (clicking on the floppy disk), and do not worry if you make any mistake, in the upper left corner you can click on "Undo".

Let's look also to the upper menu on Figure 4.20, it will help you to modify the frames and themes, and to add new elements. It is very intuitive, just a matter of clicking and marking ideas. Circular frames are usually very helpful to focus main ideas. The zoom is very important in Prezi to create a path for the connection of ideas. However, this is not the end, this is just an iteration, you can write more, keep playing with the elements in the Prezi, and adapt the focus and the zoom until you have the presentation ready. Moreover, make sure that there will be Internet connection when you want to present on-line, or that you have saved everything needed to show the presentation on a different computer (try it before the day of the presentation!). 
Third step. Show the Prezi presentation. You can do that by clicking on the blue button "Present". Prezi can automatically advance the presentation from one frame to another, or you can click when you want to move in the Prezi (backward or forward). It is also possible to show the presentation in "Full Screen" format, and my advice is that you always rehearse the presentation, because sometimes if the Prezi goes too fast and has too many movements, some people could feel a little dizzy..., trust me, you do not want that! So, show your presentation first to a few people, to get their opinion, before considering that you have it finished. As always, explore the many options that Prezi offers to you and your students, and enjoy it!

\subsection{Animoto}

To finish with the tools that I want to show you in this chapter, I want to share Animoto ${ }^{38}$ with you. It is very useful for creating videos. Sometimes, you may want to ask your students for more than a project and a presentation: a video shows voice, images in movement, sounds, because we shouldn't forget we are in the multimedia era! I hope that you are interested in learning more about Animoto. I find it fascinating. Please, join me in a step-by-step exercise to create a video with Animoto. But, first things first, I suppose you already realized that you'll need an account to use Animoto. The setup process is very easy; just fill in the form by clicking on the "Sign up" link at the upper right corner of the Animoto welcome page, as shown in Figure 4.22. Later, you will need your details to log in (it is just next to the "Sign up" button).

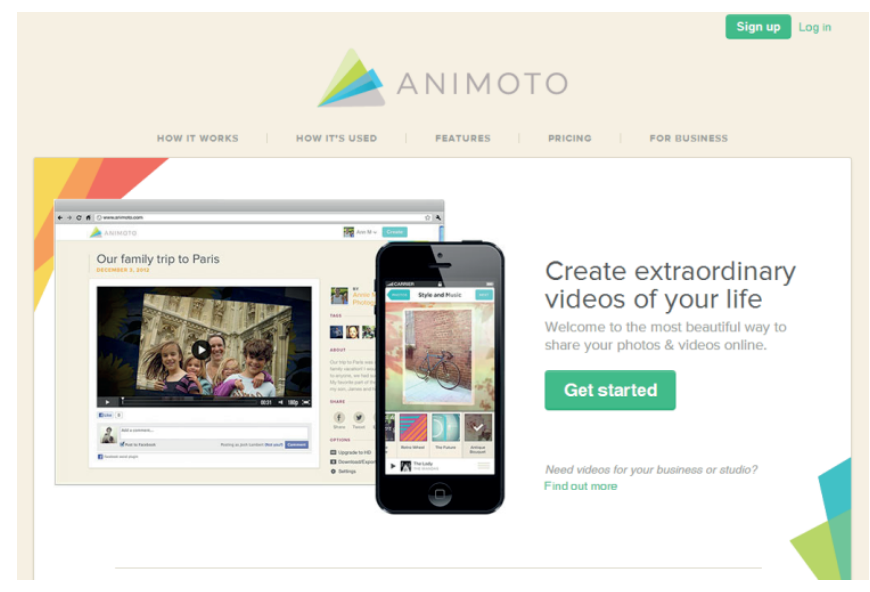

Figure 4.22: Animoto welcome webpage

38 http://animoto.com/ 
First step. Choose a style. When you have your account, you just need to click on the "Create video" button, and choose a style to determine the look and feel of your video. The easy thing here is the computer part, just to click on the style...the difficult part is to choose which one! There are many styles, and you can preview them before you choose. It is important to highlight that in the preview version, the videos are limited to 50 seconds. However, you can create small videos and later connect them with a video editing tool such as Windows Movie Maker or VideoPad Editor (the first is free if you have Windows, and the second is always free!). It is always a better idea when generating multimedia material to create many small videos, instead of one very long one.

Second step. Get your photos and videos. To create a video you need resources, just as you did when creating an activity in JClic. You can upload them from your computer, or from an external source such as a social network. Either way, take care that you have all the rights to use the material! Similarly, you should upload some music as background for the video, and even voices if you want to add some dialogue.

Third step. To edit the video. Figure 4.23 shows the editing menu of Animoto.

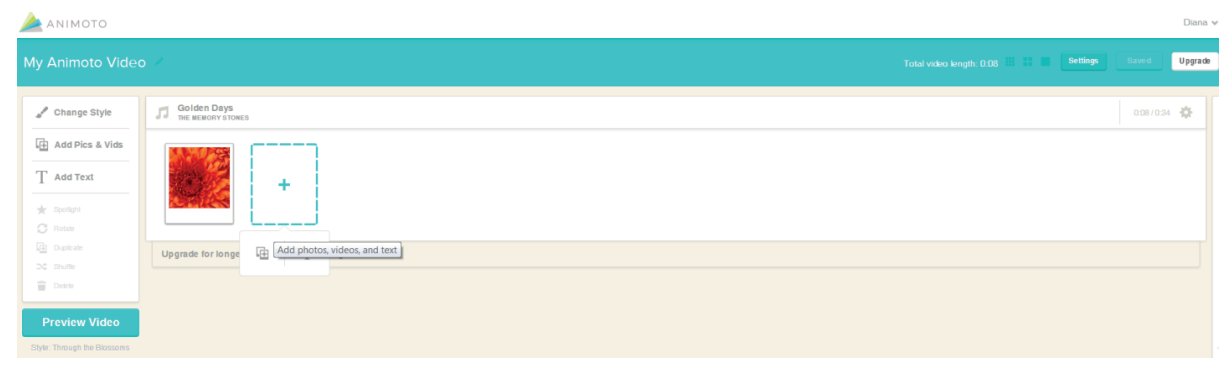

Figure 4.23: Animoto video editing page

As you can see it is pretty intuitive. You click on the element that you want to modify. For instance, if you want to change the music you click on the title of the song (in this case "Golden Days"). If you want to change an element (like the flower) you click on it, and on the left hand menu you can delete it, rotate it, duplicate it, and even give it more time in the spotlight, time so that people can enjoy the image longer in the video. You can also add more images, videos and texts by clicking on the "+" card.

Later, you can modify the sequence by moving the cards into the order that you prefer. Moreover, you can preview the video at any time by clicking on the "Preview video" button on the left, and you can change the general settings on the "Settings" button in the upper right corner. The preview will be in low resolution so that it takes less time to produce. As I mentioned, it's not the same as creating a report or 
a presentation, multimedia material consumes many more computer resources. This is why the length of the video and its quality are so important. The longer, and higher quality, the video is, the more time your computer will need to generate it. Also, viewers will need to have a better internet connection to watch your video on-line if it is long and of high quality!

Fourth step. To show and share the video. As you can see in Figure 4.24, Animoto also has a page to show your video on-line and it gives you a link to share with your friends by mail or using social networks such as Facebook. People can start commenting on your video, as soon as they have the link so make sure you have it finished before you share it, unless you want some ideas to continue your editing! You can also export the video created to other platforms such as YouTube, SmugMug or Vimeo if you have account on those platforms, but it may require 20-30 minutes for the encoding. Please, keep exploring the many possibilities that Animoto offers to you and your students, and enjoy it!

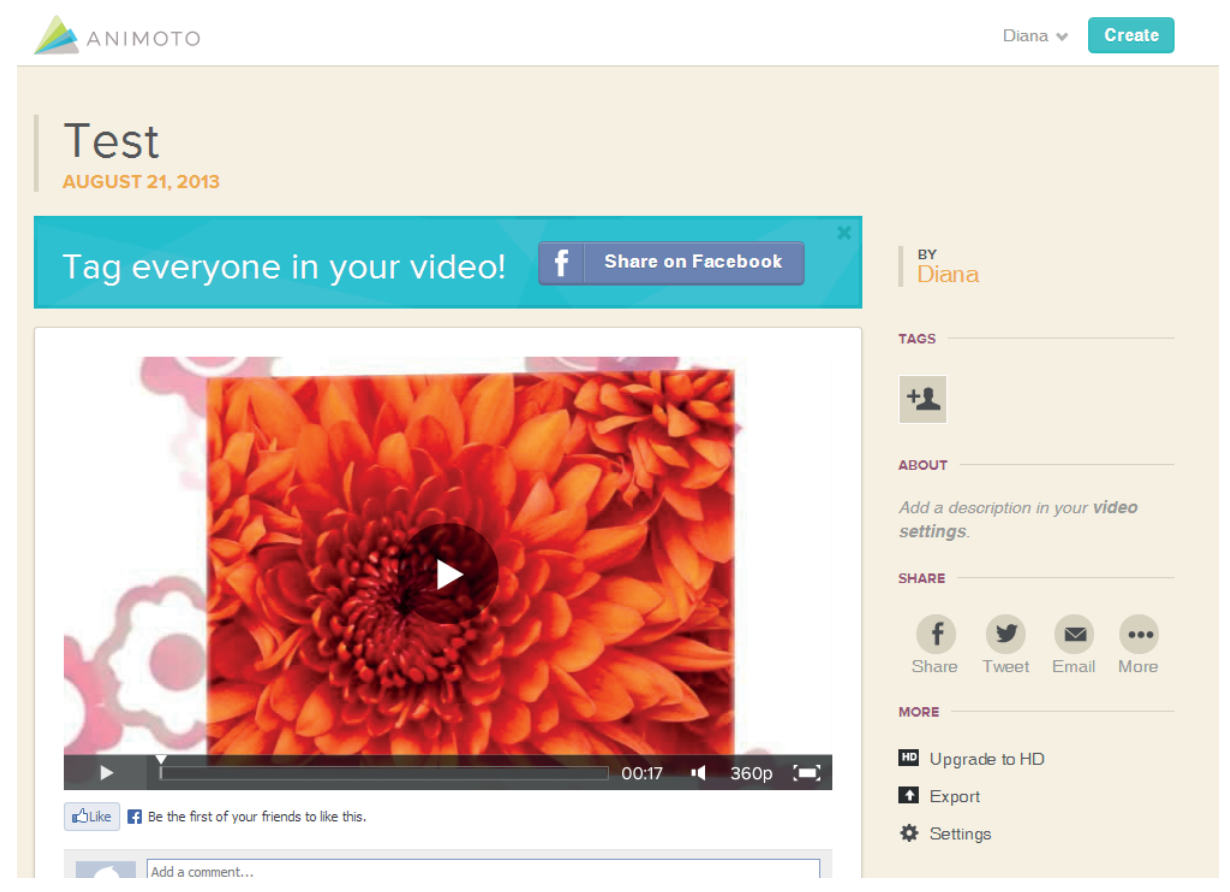

Figure 4.24: Animoto show video page 


\subsection{Exercises}

Let's do several exercises to practise the content of this chapter. Remember that all exercises are solved at the end of the chapter, but do not read the solutions until you have tried to solve the exercises on your own first! It is also possible that you'll find different answers to these questions; in that case, please tell us about them on the book's website.

1. Use Delicious to find 5 webpages about the use of ITC for Education. Save them as bookmarks in your account and tag them, so that they can be useful for other people too.

2. Use Webnotes to annotate the 5 webpages saved in Delicious to generate a brief report.

3. Find a Prezi made by other people about the use of ITC in Education (make sure that the author has given permission to reuse the Prezi), and modify it so that it has a new frame with a new idea that you want to add.

4. Create an Animoto video with the style "Confetti" to celebrate that you have finished the exercises of Chapter 4. So, the first frame in your video should show "Time to celebrate!", and a second and last frame containing some image that you have, or find on the Internet (with permission to use), of celebration. Share the video on the book's website so that other readers can watch it!

\subsection{Solved Exercises}

1) First of all, we need to log into Delicious. If you have followed the instructions in Section 4.3 you should already have a username and password. Don't worry if you have forgotten the password. You can recover it when you click "Sign in". At the bottom there is a message telling you that if you have forgotten it (it happens to many people!) Delicious will send you an email with the information to your registered mail account. Remember that if you do not already have a Delicious account, it is now an excellent time to click on the "Create an account" link to get one!

When you see the main page of Delicious, remember to use the "search" engine at the top, you can use as key words: ITC, education. The results webpage may contain links to pages that are not related to the topic, discard them! Look at the results provided to choose five good bookmarks (i.e. this is collective intelligence). You can see my bookmarks at https://delicious.com/perezmarin_7. Please, share on the book's website your own bookmarks, they may be really interesting!

Do not forget to sign out when you have finished, by clicking on the "Sign out" option in the menu associated to your username (on the upper right corner). 
2) I confess, I want to make you practice sign out and log in. So, let's log in again in Delicious, and click on the webpages that you have saved, because it is now time to annotate them and generate a report in Webnotes. Remember to click on the red tack if you have downloaded the Google Chrome extension of Webnotes, or click on "Annotate" in the Webnotes menu to start highlighting and saving comments. I also advise you to create a folder "ICT for Education" to save all these annotations (you can do that by clicking on the "Folder" option of the menu $\rightarrow$ "Create a new folder"). You should have a report similar to the one shown in Figure 4.25.

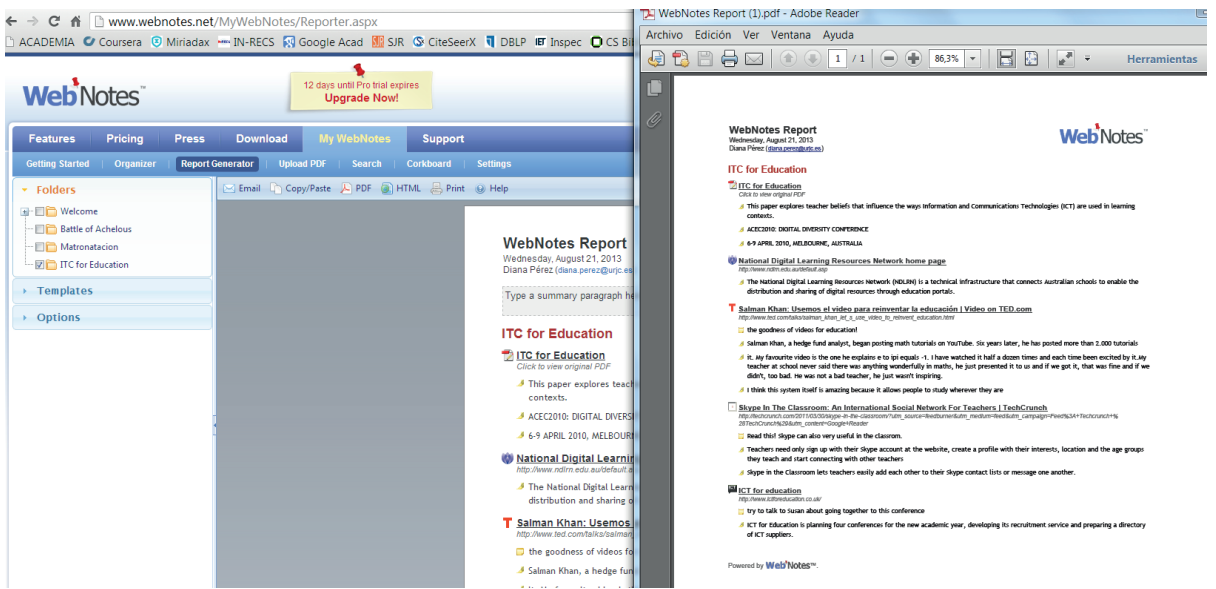

Figure 4.25: Sample Webnotes “ITC for Education” report generated

Do not forget to download the PDF by clicking on the "PDF" icon and save it on your computer as you will need it for the next exercise!

3) If you go to the main page of Prezi, you can click on "Explore" and see presentations made by other people. I like the presentation made by Cali Pratt "Welcome back to School”. You can watch it at http://prezi.com/6wubchu75u9p/welcome-back-toschool/. Cali has marked it as "Public and reusable". So, I am going to use it to add the idea that you can use ITC for Education. First of all, I make a copy, so that I do not modify the original Prezi presentation. To do that, I need to log in. As in the first exercise, if you have forgotten your password, do not worry because Prezi is also able to send you a message with your user account information. Once you have made the copy, the presentation appears next to the one we created in Section 4.4. So, we can click on the new presentation to edit it, and add the new frame by clicking on the "Frames \& Arrows" option at the top. You will have something similar to the page shown in Figure 4.26. 


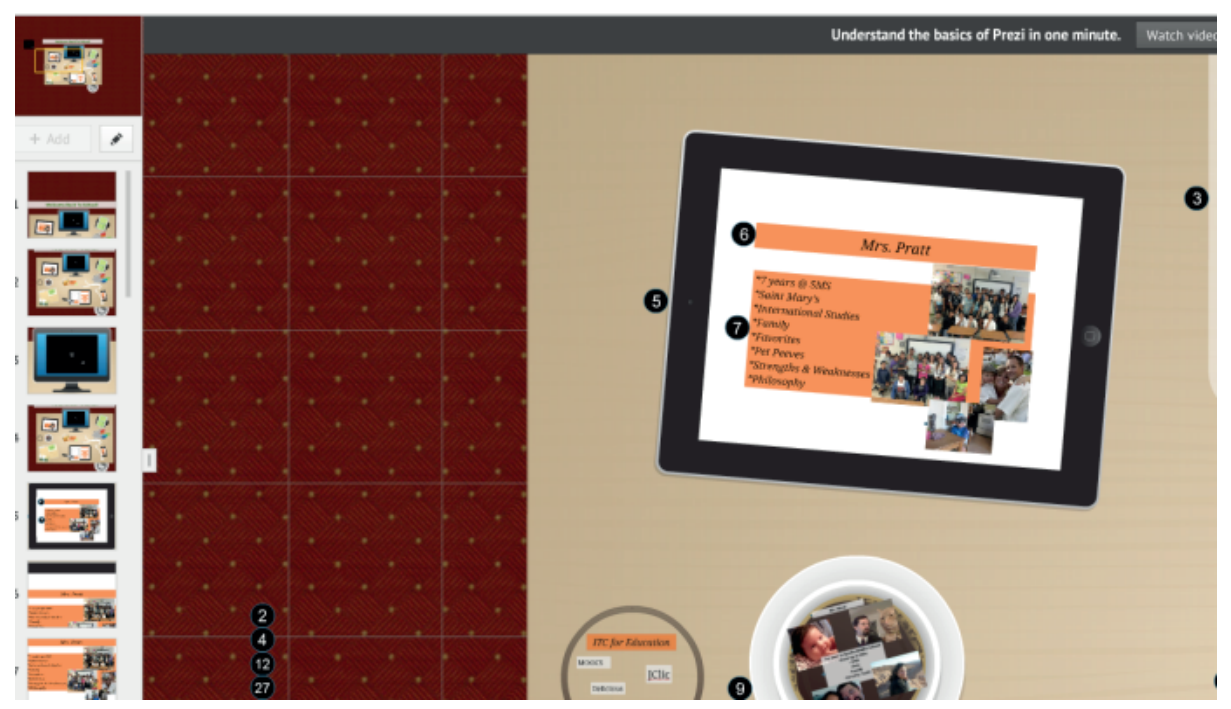

Figure 4.26: Adding ideas in a Prezi presentation

4) OK, so first of all, remember your user account details to log into the Animoto webpage. Next, you need to click on the "Create" button and choose the "Confetti" style $\rightarrow$ make a video of 30 seconds for free. Once you are in the editing page then you can add a text frame with the text "Time to celebrate!" and add a second frame with an image of celebration. I chose from the Animoto "Image library", in the category "Birthday", the image with the confetti, but you can choose any other image that you like. Once you have completed all these steps, you can preview your video by clicking on the "Preview video" button (on the left). Remember that working with videos is a time consuming task, so be patient while the video is processed. Once you have a link, please share it on the book's website. As an example, I've shared mine:

http://animoto.com/play/IvqwEqmYNvFIX6Ek01T40Q

You can edit a video after having processed it, by clicking on the Animoto webpage, in your user account $\rightarrow$ "My videos", and clicking on the little wheel under the video. 